\title{
Variational Optical Flow Estimation for Images with Spectral and Photometric Sensor Diversity
}

\author{
Tomas Bengtsson ${ }^{1}$, Tomas McKelvey ${ }^{1}$ and Konstantin Lindström ${ }^{2}$ \\ ${ }^{1}$ Department of Signals and Systems, Chalmers University of Technology, Sweden \\ ${ }^{2}$ Volvo Cars AB, Sweden
}

\begin{abstract}
Motion estimation of objects in image sequences is an essential computer vision task. To this end, optical flow methods compute pixel-level motion, with the purpose of providing low-level input to higher-level algorithms and applications. Robust flow estimation is crucial for the success of applications, which in turn depends on the quality of the captured image data. This work explores the use of sensor diversity in the image data within a framework for variational optical flow. In particular, a custom image sensor setup intended for vehicle applications is tested. Experimental results demonstrate the improved flow estimation performance when IR sensitivity or flash illumination is added to the system.
\end{abstract}

Keywords: Optical flow, Sensor diversity, Infrared, Variational framework

\section{INTRODUCTION}

Optical flow estimation is the task of finding pixel-level displacements between two images in a sequence [1]. It is an essential computer vision technique that has numerous applications, for example in vehicle active safety systems and medical imaging technology. An issue for optical flow estimation is poorly contrasted image data, which results in corrupted flow estimates. In turn, this causes problems for e.g. a tracker of an application system [2]. For active safety systems it is reported that poorly contrasted image data, especially for challenging conditions such as night time or fog, is a limiting factor for classification and tracking performance of pedestrians and other objects of interest [3]. Lack of contrasts between objects may occur for different reasons; either the dynamic range of the imaged scene may exceed that of the image sensor, which causes saturation, or the imaged objects may fundamentally have a low contrast in the spectral bands of the sensor, e.g. if a person wears clothing of a similar color to the background. These scenarios may be remedied by using multiple exposure durations or by capturing data from additional spectral bands, such as Infrared (IR) data, respectively. In this work, a general optical flow formulation for vector-valued images is presented, which naturally encompasses photometric- and spectral diversity in the image data.

To estimate optical flow, a common approach is to cast the problem in a variational framework $[4,5]$. Thus, the image pair under analysis is assumed to be time-samples of a continuous image intensity function $I(x, y, t)$. The flow estimation process then corresponds to minimizing a cost functional, which is typically derived based on the Brightness Constancy Assumption (BCA). The BCA states that a given image location has the same brightness at its new location in a subsequent time instance, that is

$$
I(x+u(x, y), y+v(x, y), t+1)-I(x, y, t)=0,
$$

where $(x, y)$ are the image coordinates, $t, t+1$ are the sampling instances (using general sampling instances simply requires that the flow components are scaled according to the time difference) and the image flow field is modeled by $u(x, y)$ and $v(x, y)$ as the translation in $x$ and $y$ coordinates respectively. This is often denoted as the optical flow data constraint. In the estimation process, the two-dimensional flow field estimate $(u, v)$ should keep deviations from the assumption as small as possible. If the image data is scalar, e.g. grayscale intensity, it is clear that the data constraint is insufficient to formulate a minimization problem with a unique solution, as there are two flow components per data point. An additional condition must be added to constrain the solution. The common choice is to add a global regularization term that enforces a (piecewise) smooth solution. Even for multichannel images, such as three channel color images where the BCA is applied to each individual channel, the regularization term is still necessary because typically certain image regions, such as the interior of homogenous objects, lack texture in all channels simultaneously.

Sixth International Conference on Graphic and Image Processing (ICGIP 2014),

edited by Yulin Wang, Xudong Jiang, David Zhang, Proc. of SPIE Vol. 9443, 94431U

(c) 2015 SPIE $\cdot$ CCC code: 0277-786X/15/\$18 · doi: 10.1117/12.2180109 
The variational formulation for optical flow was first adopted by Horn and Schunk [4]. It provides a rigid framework for estimating non-integer, subpixel-valued flow, and theoretically justifies image warping as part of the algorithm [5]. Furthermore, there are solid numerical methods that solve the corresponding minimization problem at or close to realtime [6]. In the initial Horn-Schunk method, the BCA (1) was directly linearized to $I_{x} u+I_{y} v+I_{t}=0$ in the cost functional formulation, where $I_{x}, I_{y}$ and $I_{t}$ denote the partial derivatives of the intensity function. Because the BCA in its original form is highly non-monotonic in the displacements $(u, v)$, the linearization makes the solution prone to get trapped in local minima, disabling the correct estimation of large actual displacements. More recent variational optical flow methods thus avoid direct linearization of the BCA (around a zero displacement), and instead successively linearize the problem about the current flow estimate in the numerical solution scheme [2,5,7]. Quadratic relaxation of the cost functional by introducing auxiliary flow variables leads to another implementation method, where the minimization is divided into two steps which can each be solved effectively [6,8]. High resolution images, or cases in general that contain large displacements, benefit from a coarse-to-fine estimation strategy, in which images are re-sampled to different spatial resolutions [9]. In modern methods, robust regularization is used in terms of anisotropic smoothing along but not across edges [7], or L1-based total variation [10]. The addition of temporal regularization (rather than only spatial) or an image feature matching term are recent advances of optical flow estimation methods that have shown promising results, but are outside of the current scope [11, 12]. For image data from non-controlled environments, it is reported that structure-texture decomposition as well as alternative color spaces can be helpful to reduce the effects of natural illumination changes and shadows [13].

A few results have been reported on optical flow on visual and IR data jointly, whereas there is additional work on optical flow from a lone IR camera, e.g. for automated human detection [14]. Our interest, however, is on methods that make use of the spectral diversity by treating visual and IR data jointly. One such approach found in the literature is to compute the flow from each modality separately but use a cross-regularization term and a merging strategy to couple the estimation process [15]. In contrast, this paper proposes to include all data in a single cost functional, in order to promote proper flow estimates of objects that only appear in one image channel, due to poor exposure or lack of contrasts in the remaining channels. The sensor setup at our disposal is presented next.

\subsection{Joint Visual and Infrared Image Sensor Setup}

A custom camera sensor system is set up in a test vehicle in order to investigate its usage. The sensor array is designed as shown in Figure 1 (a). One fourth of the sensor pixel elements, denoted ' $\mathrm{V}$ ' in the figure, are sensitive to visual light, covering the red, green and blue spectra and thus produce a single grayscale value each. The remaining pixels, denoted 'W', have a wider spectral sensitivity, which also includes frequencies of the near-IR region in addition to the visual spectrum.

For every other exposure, the system alternates between using the headlights of the vehicle to flash the scene (with IR light) during the image capture, as depicted in Figure 1 (b). The multi-channel images $\boldsymbol{I}_{1}$ and $\boldsymbol{I}_{2}$ each consist of data from one exposure with and one without flash and the data from each exposure is demosaiced into two separate channels with different spectral sensitivities. The remainder of the paper is as follows. Section 2 presents the variational optical flow method employed, Section 3 discusses experimental results and, finally, Section 4 gives some concluding remarks.

\section{OPTICAL FLOW ESTIMATION IN A VARIATIONAL FRAMEWORK}

Variational optical flow estimation corresponds to minimizing a cost functional which in general can be written in the form

$$
E(u, v)=\iint_{\Omega}\left(F_{D a t a}+\alpha F_{R e g}\right) d x d y
$$

where $\Omega \in \mathbb{R}^{2}$ is the image domain, $F_{\text {Data }}$ is a term that penalizes deviations from the adopted data constraint, most often, including here, based on the BCA (1), $F_{\text {Reg }}$ enforces some regularity condition that can be seen as prior information on the flow and $\alpha$ is a weight parameter. From here on, consider the general case of optical flow estimation between a pair of image frames, $\boldsymbol{I}_{1}(x, y)=\boldsymbol{I}(x, y, t=0), \boldsymbol{I}_{2}(x, y)=\boldsymbol{I}(x, y, t=1)$, where the underlying image function $I=\left\{I^{1}, \ldots, I^{C}\right\}$ has a set of channels (dimensions), indexed $c=1, \ldots, C$. The vector-valued extension of the cost functional in [5], which should be minimized to estimate $(u, v)$, can be written as 

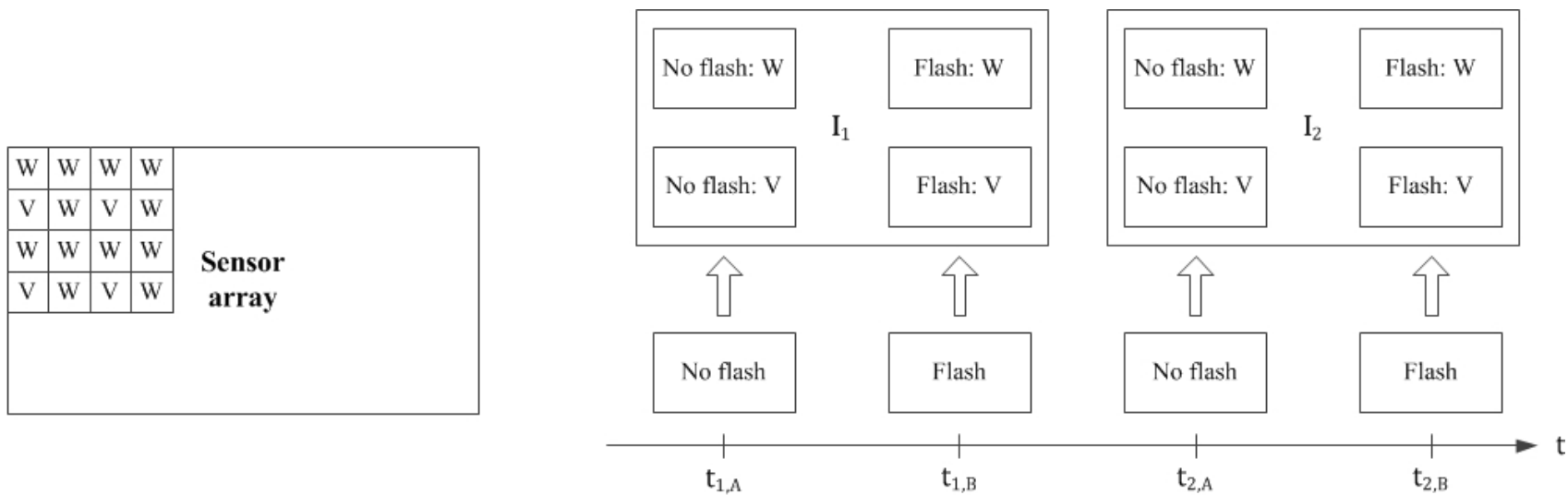

Figure 1. (a) Left: The pixel elements of the sensor array are sensitive to either the visual spectrum (V) or the wider band of visual and near-IR information (W). (b) Right: Every other frame is captured with different exposure settings. The main difference is whether or not flash illumination is used. From each exposure, the data is separated into two image channels, one with the visual data the other with wideband data. Interpolation is performed to fill in missing pixel values in both channels. Finally, $\boldsymbol{I}_{1}$ and $\boldsymbol{I}_{2}$ are each formed using data from one exposure with and one without flash.

$$
E(u, v)=\iint_{\Omega}\left(\Psi\left(\sum_{c=1}^{C} \beta_{c}\left[I_{2}^{c}(x+u, y+v)-I_{1}^{c}(x, y)\right]^{2}\right)+\alpha \Psi\left(u_{x}^{2}+u_{y}^{2}+v_{x}^{2}+v_{y}^{2}\right)\right) d x d y
$$

where the $\beta_{c}$ are weight constants and $\Psi\left(z^{2}\right)=\left(z^{2}+\varepsilon^{2}\right)^{1 / 2}, \varepsilon=10^{-3}$, is a convex L1-norm approximation which promotes edge-preserving spatial smoothing by expressing the total variation of the flow vector [16], and also makes the method more robust to outliers in the data.

According to the calculus of variations, a minima of (3) is found when the first variation with respect to the unknown functions are equal to zero, i.e. when $\delta E / \delta u=0, \delta E / \delta v=0$. These are the familiar Euler-Lagrange (E-L) equations, and can be stated in general form according to

$$
\frac{\delta E}{\delta u}=0 \Leftrightarrow \sum_{n} D_{n}^{T}\left(\frac{\partial F}{\partial D_{n} u}\right)=0
$$

and analogously for $\delta E / \delta v=0$ [17]. Here, $F\left(u, u_{x}, u_{y}, v, v_{x}, v_{y}\right) \equiv F_{D a t a}+\alpha F_{R e g}$ is the integrand of (3), and the derivative operator $D_{n}$ represents $\left\{D_{0} u, D_{1} u, D_{2} u\right\}=\left\{u, u_{x}, u_{y}\right\}=\{u,(\partial / \partial x) u,(\partial / \partial y) u\}$. The transposed $D_{n}^{T}=-D_{n}$ for derivatives $D_{n}$ of odd order and $D_{n}^{T}=D_{n}$ otherwise. Introducing the abbreviations

$$
\begin{aligned}
& I_{z}^{c}=I_{2}^{c}(x+u, y+v)-I_{1}^{c}(x, y) \\
& I_{x}^{c}=\partial I_{2}^{c}(x+u, y+v) / \partial x \\
& I_{y}^{c}=\partial I_{2}^{c}(x+u, y+v) / \partial y
\end{aligned}
$$

for notational brevity in the derivation of the method, evaluating the right hand side of (4) yields

$$
\begin{aligned}
& \Psi^{\prime}\left(\sum_{c=1}^{C} \beta_{c}\left[I_{z}^{c}\right]^{2}\right) \cdot \frac{\partial}{\partial u}\left(\sum_{c=1}^{C} \beta_{c}\left[I_{z}^{c}\right]^{2}\right)-\alpha \frac{\partial}{\partial x}\left(\Psi^{\prime}\left(u_{x}^{2}+u_{y}^{2}+v_{x}^{2}+v_{y}^{2}\right) \cdot 2 u_{x}\right) \\
& -\alpha \frac{\partial}{\partial y}\left(\Psi^{\prime}\left(u_{x}^{2}+u_{y}^{2}+v_{x}^{2}+v_{y}^{2}\right) \cdot 2 u_{y}\right)=0,
\end{aligned}
$$


where $\Psi^{\prime}\left(z^{2}\right)=(1 / 2)\left(z^{2}+\varepsilon^{2}\right)^{-1 / 2}$. The flow functions $(u, v)$ are contained within $I_{z}^{c}$ and the flow that satisfies $(6)$ as well as $\delta E / \delta v=0$ is computed iteratively in a numerical scheme with successive linearizations (linearization of the data constraint (1) directly in the cost expression (3) is thus avoided [5]). To this end, the unknowns are split into $u^{k+1}=u^{k}+d u^{k}, v^{k+1}=v^{k}+d v^{k}$, the estimates at the previous iteration $k$ and the increments, that are the new minimization variables. Insertion of $u \rightarrow u^{k+1}, v \rightarrow v^{k+1}$ and the linearized $I_{z}^{c} \rightarrow\left(I_{z}^{c}\right)^{k}+\left(I_{x}^{c}\right)^{k} d u^{k}+\left(I_{y}^{c}\right)^{k} d v^{k}$ into (6) gets us halfway to a linear formulation. The remaining non-linearity, $\Psi^{\prime}$, is treated by adding an inner iteration loop over $l$, such that the update equation of the E-L equation w.r.t. $u$ becomes

$$
\begin{aligned}
& \Psi^{\prime}\left(\sum_{c=1}^{C} \beta_{c}\left[\left(I_{z}^{c}\right)^{k}+\left(I_{x}^{c}\right)^{k} d u^{k, l}+\left(I_{y}^{c}\right)^{k} d v^{k, l}\right]^{2}\right) \cdot\left(\sum_{c=1}^{C} \beta_{c} I_{x}^{c}\left[\left(I_{z}^{c}\right)^{k}+\left(I_{x}^{c}\right)^{k} d u^{k, l+1}+\left(I_{y}^{c}\right)^{k} d v^{k, l+1}\right]\right)- \\
& -\alpha \frac{\partial}{\partial x}\left(\Psi^{\prime}\left(\left(u^{k}+d u^{k, l}\right)_{x}^{2}+\left(u^{k}+d u^{k, l}\right)_{y}^{2}+\left(v^{k}+d v^{k, l}\right)_{x}^{2}+\left(v^{k}+d v^{k, l}\right)_{y}^{2}\right) \cdot\left(u^{k}+d u^{k, l+1}\right)_{x}\right)- \\
& -\alpha \frac{\partial}{\partial y}\left(\Psi^{\prime}\left(\left(u^{k}+d u^{k, l}\right)_{x}^{2}+\left(u^{k}+d u^{k, l}\right)_{y}^{2}+\left(v^{k}+d v^{k, l}\right)_{x}^{2}+\left(v^{k}+d v^{k, l}\right)_{y}^{2}\right) \cdot\left(u^{k}+d u^{k, l+1}\right)_{y}\right)=0 \Leftrightarrow \\
& \Leftrightarrow C_{1} \cdot\left(\sum_{c=1}^{C} \beta_{c} I_{x}^{c}\left[\left(I_{z}^{c}\right)^{k}+\left(I_{x}^{c}\right)^{k} d u^{k, l+1}+\left(I_{y}^{c}\right)^{k} d v^{k, l+1}\right]\right)-\alpha \operatorname{div}\left(C_{2} \cdot \nabla\left(u^{k}+d u^{k, l+1}\right)\right)=0,
\end{aligned}
$$

where $\nabla$ is the gradient operator and div is the divergence operator. Notice that the equation is linear in the flow increments $\left(d u^{k, l+1}, d v^{k, l+1}\right)$, because the arguments of $\Psi^{\prime}(\cdot)^{k, l}$ only contain data from the previous iteration step and thus are constant w.r.t. the current update, as highlighted by the introduction of the fixed functions $C_{1}(x, y)$ and $C_{2}(x, y)$ (the latter is the $\Psi^{\prime}(\cdot)^{k, l}$-term of the smoothing regularization). Similarly, the update equation of the E-L equation w.r.t. $v$ is

$$
C_{1} \cdot\left(\sum_{c=1}^{C} \beta_{c} I_{y}^{c}\left[\left(I_{z}^{c}\right)^{k}+\left(I_{x}^{c}\right)^{k} d u^{k, l+1}+\left(I_{y}^{c}\right)^{k} d v^{k, l+1}\right]\right)-\alpha \operatorname{div}\left(C_{2} \cdot \nabla\left(v^{k}+d v^{k, l+1}\right)\right)=0
$$

In the numerical implementation of the estimation procedure, the flow increments are computed on the discrete pixel grid of the images. Re-arranging the terms in (7), (8) leads to a system of linear equations, $\boldsymbol{A}^{k, l}\left[\left(\boldsymbol{d} \boldsymbol{u}^{k, l+1}\right)^{T},\left(\boldsymbol{d} v^{k, l+1}\right)^{T}\right]^{T}=\boldsymbol{b}^{k, l}$ (formed by vectorizing the image coordinates). Discrete approximations of the derivative terms are used to construct $\boldsymbol{A}^{k, l}, \boldsymbol{b}^{k, l}$. The pseudo-algorithm for the full iterative scheme is given in Table 1.

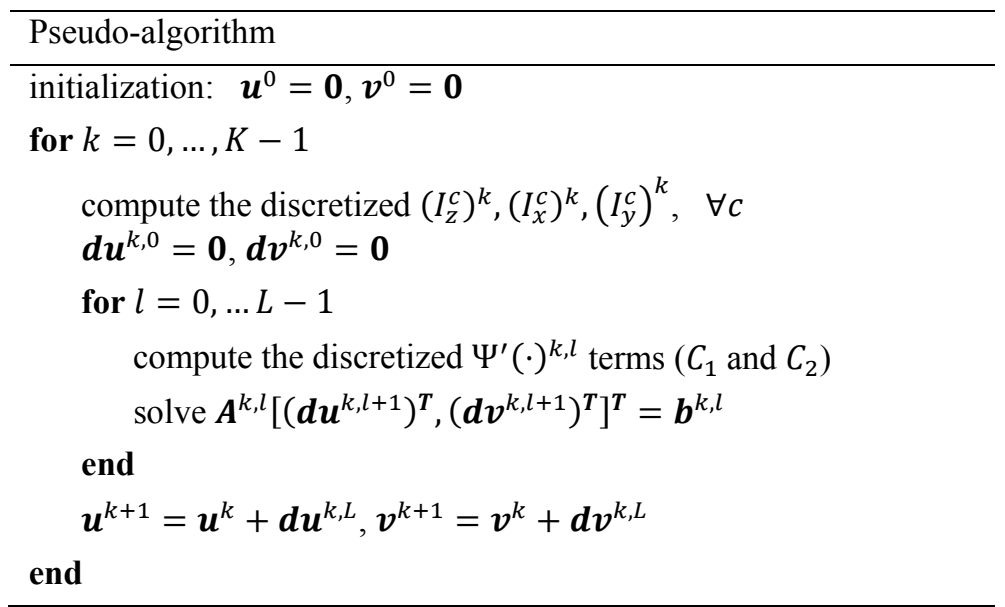

Table 1. Optical flow estimation performed by minimizing a variational cost functional on a discretized numerical grid. 


\section{EXPERIMENTAL RESULTS AND DISCUSSION}

This section presents two experimental results. As discrete derivatives, the convolution kernels $[0.5,0,-0.5]$ and $[0.5,0,-0.5]^{T}$ are used. The divergence terms in (7) and (8) are implemented as the generalized Laplacian in Appendix A of [18]. For an in-depth discussion on an alternative and often adopted numerical implementation, refer to [19]. Furthermore, bicubic interpolation is used to warp image data, i.e. to evaluate $I_{2}^{c}(x+u, y+v)$ in (5) on the discrete pixel grid of $I_{1}^{c}(x, y)$, as $(u, v)$ are generally non-integer values. All image channels are weighted equally by setting $\beta_{c}=1, \forall c$, and a good tradeoff between the data term and the spatial regularization, stabilizing the solution while avoiding overmoothing, is achieved experimentally with $\alpha=0.1$, except where otherwise stated. (the performance is not particularly sensitive to the exact parameter choice). The number of outer and inner iterations are $K=5, L=5$ respectively for both experiments, proven sufficient for convergence. Unfortunately, no ground truth flow is available for evaluation in the experiments.

Experiment 1 is performed on images from the OTCBVS Benchmark Dataset Collection ${ }^{*}$, in order to test the general vector-valued optical flow method on public data. Frames number 1 and 7, of resolution $190 \times 270$ pixels, from Dataset 03: OSU Color-Thermal Database are used. The image frames consist of (gamma encoded) R',G',B' color data as well as thermal IR data from a separate camera that has been registered spatially by a (global) homography. Figure 2 displays the original images on the top row and 4 different estimated flow results on the bottom row, see the encoding chart in Figure 3. The color encodes angular direction of the flow and the intensity encodes the magnitude. In the leftmost result in Figure 2, where the R', G',B' channels were used in the flow estimation, there appears to be significant image flow caused by the shadow from the house (and the persons). In the next subfigure, the R', G', $\mathrm{B}^{\prime}$ data was transformed to the $\mathrm{Y}^{\prime}, \mathrm{Cb}$ ',Cr' color space, the luma-channel $\mathrm{Y}^{\prime}$ removed and the data channel from the IR camera added to the chroma components $\mathrm{Cb}$ ',Cr' of input images. Third from the left is the result of using only the IR channel, which produces somewhat noisy estimates, and thus supports the inclusion of the chroma channels. The final result is from only the $\mathrm{Cb}^{\prime}, \mathrm{Cr}$ ' channels, for which a lower $\alpha=0.01$ has been used due to lower overall contrasts in those channels.

Experiment 2 is performed on data captured by the prototype camera setup described in Section 1.1. Raw data of image resolution $360 \times 640$ is captured from a night-drive in an animal park. Each image $\boldsymbol{I}_{f}, f=\{1,2\}$ is considered to consist of four image channels, although the first two are captured at time $t_{f, A}$ and the other two at $t_{f, B}$, the difference being that the headlights flash with IR light during the second exposure. Here, simply assume $t_{1, A}=t_{1, B}=0$ and $t_{2, A}=t_{2, B}=1$. The four channels of frame 1 are visualized on the top row of Figure 3, and one channel from frame 2 at the bottom left, processed according to $I_{f}^{c}=\left(\left[\Delta t_{c} X_{f}^{B P_{c}}\right] / \max _{c}\right)^{1 / \gamma}$, where $X_{f}^{B P_{c}}$ denotes sensor illuminance through the bandpass filter structure with visual (V) or wideband (W) spectral sensitivities (disregarding the demosaicing in the model). Each channel $c$ has a corresponding exposure duration $\Delta t_{c}$. Furthermore, for visualization in the figure, the image data is normalized (contrast stretched) so that the maximum value is 1 , and then gamma encoded with $\gamma=2.2$. Here, the $\Delta t_{c}$ only differ by some percentage between the flash and the non-flash channels, but they could in general be set freely to allow a higher dynamic range. Instead, the flash provides a similar effect here.

In this experiment, a coarse-to-fine estimation strategy is used, with $S=13$ scale levels. Level $s=1$ is re-sampled by a factor $0.9^{S-s} \approx 0.28$ from the original resolution. For each scale, the algorithm in Table 1 is run, although for $s>1, \boldsymbol{u}^{0}, \boldsymbol{v}^{0}$ are assigned values corresponding to the re-scaled solution from the previous scale. Results of three flow estimations, directly on the raw data $\Delta t_{c} X_{f}^{B P_{c}}$, are shown in columns 2 to 4 of the bottom rows of Figure 3 . The circle diagram to the left displays the color encoding of the flow components. Its outer radius corresponds to a magnitude of 20 pixels. First from the left is the result from flow estimation on the two non-flash channels, with $\alpha=0.001$. Lack of contrasts in the woods results in poor estimates of too low magnitude, and some regions contain rather arbitrary flow estimates, seen as noisiness in the color encoding of the flow. The next two results, with flash images as well as with all four channels, give similar results, however using all channels gives flow estimates on the road surface that match manual inspection somewhat better. The vehicle ego-motion, as can be seen, results in flow components in the downward direction and out to the sides. The quiver plots that zoom in on the deer show equal results for all experiments (flow is only shown at every third pixel on the right sides). The leg movement of the deer is distinguishable from the dominant flow caused by the vehicle ego-motion. However, the flash channels are better suited to help detect animals at the side of the road.

\footnotetext{
* Images are courtesy of James Davis and Vinay Sharma, available at the website http://vcip1.ecen.okstate.edu/otcbvs/bench/.
} 


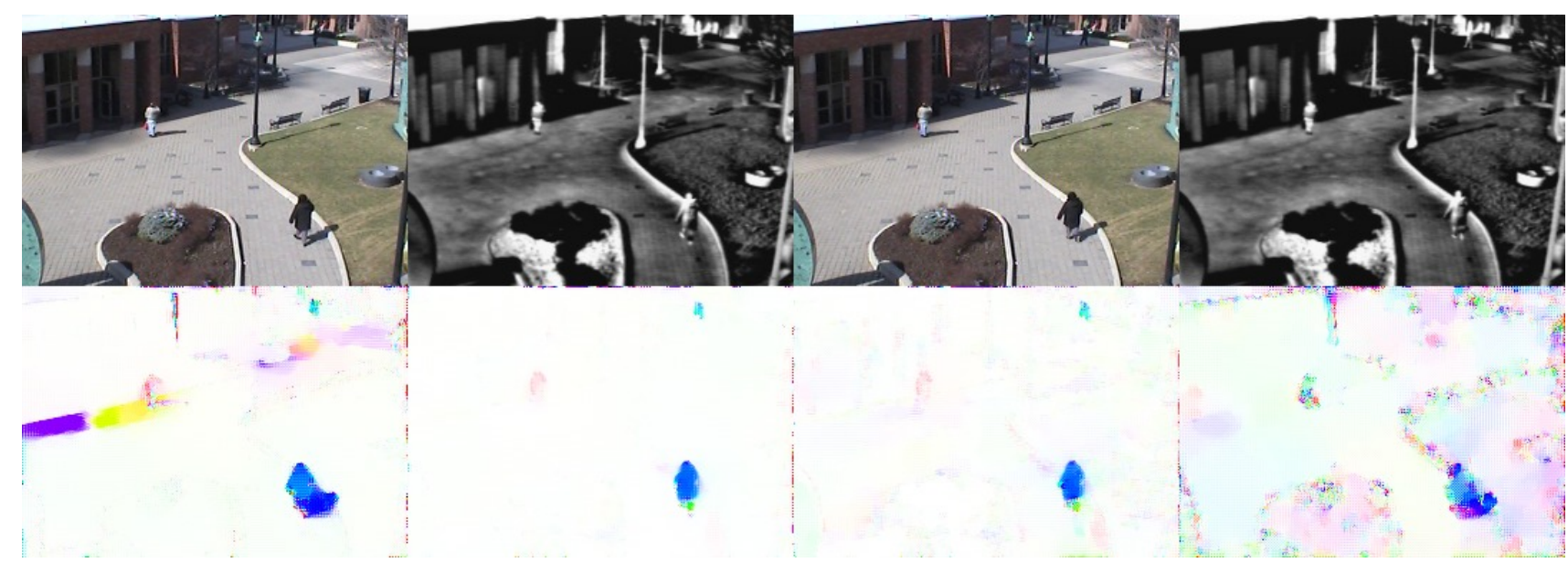

Figure 2. Top row: Frames 1 (left pair) and 7 (right pair) from the visual and IR camera respectively. Bottom row, left to right: Estimated flow results from R',G',B' image data, Cb',Cr',IR, only IR and finally from the Cb',Cr' channels. The color encoding is the same as shown in Figure 3. The flow estimates are best viewed in digital format.

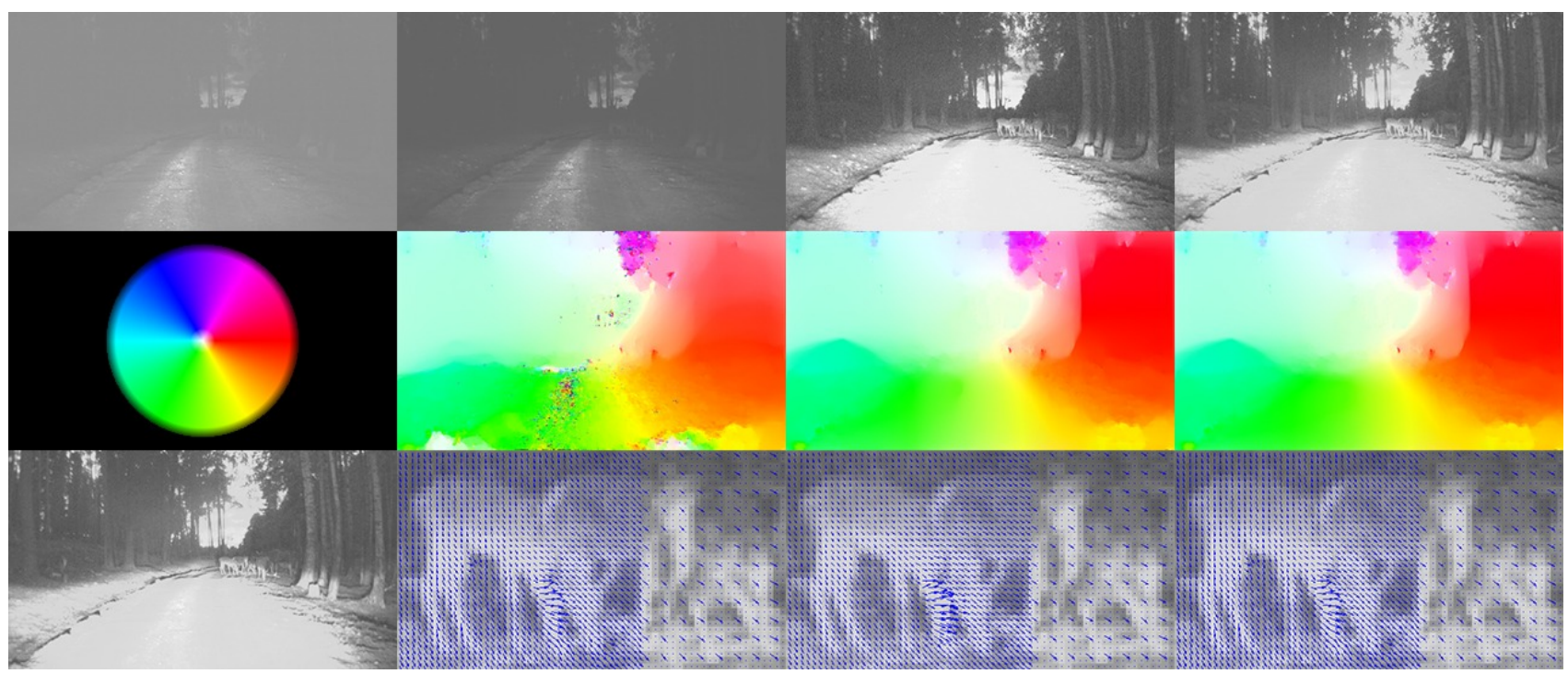

Figure 3. Top row: The four channels of frame 1, two without flash and the others with. In each pair, the right image has IRsensitivity. Bottom two rows: Visualization diagram of flow components, where the outer radius corresponds to a magnitude of 20 pixels, and example channel from frame 2. Then, in order from left to right, estimation results including corresponding zoomed-in quiver plots from the two channels without flash, the two with flash and all four channels respectively.

\section{CONCLUSIONS AND FUTURE WORK}

In this work, benefits of exploiting sensor diversity for optical flow have been discussed, and a method has been outlined in a general variational framework. In Experiment 1, a shadow region caused some artificial flow in the estimate from R', G',B' data, as previously observed in [13]. Here, the use of IR data was shown to aid the chroma channels in flow estimation by providing an alternative intensity channel to luma. For the more challenging Experiment 2, the use of the headlights to illuminate the scene improved the flow results, but the results nevertheless pointed to some weaknesses inherent in the adopted optical flow method. Depth-aware, 3-dimensional spatial regularization would yield more realistic smoothing of e.g. the road surface than regularization on the projected image plane. Local descriptor features should be considered to handle small-scale complex movement which may otherwise risk to be drowned in dominant, larger-scale motion [12]. The focus on image sequences captured with alternating exposure settings is maintained for future work, due to the demand of robust flow estimation as well as the promising initial results from this work. 


\section{REFERENCES}

[1] J. Barron, D. Fleet and S. Beauchemin, "Performance of optical flow techniques," International Journal of Computer Vision 12(1), 43-77 (1994).

[2] A. Wedel, C. Rabe, T. Vaudrey, T. Brox, U. Franke and D. Cremers, "Efficient dense scene flow from sparse or dense stereo data," Springer (2008).

[3] D. Geronimo, A. Lopez, A. Sappa and T. Graf, "Survey of pedestrian detection for advanced driver assistance systems," IEEE Transactions on Pattern Analysis and Machine Intelligence 32(7), 1239-1258 (2010).

[4] B. Horn and B. Schunk, "Determining optical flow," Artificial intelligence 17(1), 185-203 (1981).

[5] T. Brox, A. Bruhn, N. Papenberg and J. Weickert, "High accuracy optical flow estimation based on a theory for warping," Computer Vision-ECCV 2004, 25-36, Springer Berlin Heidelberg (2004).

[6] C. Zach, T. Pock and H. Bischof, "A duality based approach for realtime tv-11 optical flow," Pattern Recognition, 214-223, Springer (2007).

[7] H. Zimmer, A. Bruhn, J. Weickert, L. Valgaerts, A. Salgado, B. Rosenhahn and H. Seidel, "Complementary optical flow," Energy minimization methods in computer vision and pattern recognition, 207-220, Springer (2009).

[8] A. Wedel and D. Cremers, "Stereo scene flow for 3D motion analysis", Springer (2011).

[9] E. Memin and P. Perez, "A multigrid approach for hierarchical motion estimation," Computer Vision, 1998. 933938, IEEE (1998).

[10] A. Chambolle and P. Lions, "Image recovery via total variation minimization and related problems," Numerische Matematik 76(2), 167-188 (1997).

[11] S. Volz, A. Bruhn, L. Valgaerts and H. Zimmer, "Modeling temporal cohnerence for optical flow," in IEEE conference on Computer Vision (ICCV), 1116-1123, IEEE (2011).

[12] T. Brox and J. Malik, "Large displacement optical flow: descriptor matching in variational motion esetimation," IEEE Transactions on Pattern Analysis and Machine Intelligence 33(3), 500-513 (2011).

[13] C. Vogel, S. Roth and K. Schindler, "An evaluation of data costs for optical flow," Pattern Recognition, 343-353, Springer (2013).

[14] A. Fernández-Caballero, J. Castillo, J. Martinez-Cantos and R. Martinez-Tomás, "Optical flow or image subtraction in human detection from infrared camera on mobile robot," Robotics and Autonomous Systems 58(12), 1273-1281 (2010).

[15] H. Man, R. Holt, J. Wang, R. Martini, R. Netravali and I. Mukherjee, "A new optical flow estimation method in joint eo/ir video surveillance," Defense and Security Symposium, International Society for Optics and Photonics (2007).

[16] P. Blomgren and T. Chan, "Color tv: total variation methods for restoration of vector-valued images," IEEE Transactions on Image Processing 7(3), 304-309 (1998).

[17] G. Strang, "Computational Science and Engineering", Section 7.2, Wellesley-Cambridge Press (2007).

[18] C. Liu, "Beyond pixels: exploring new representations and applications for motion analysis," PhD thesis, Massachusetts Institute of Technology (2009). http://people.csail.mit.edu/celiu/Thesis/CePhDThesis.pdf

[19] P. Perona and J. Malik, "Scale-space and edge detection using anisotropic diffusion," IEEE Transactions on Pattern Analysis and Machine Intelligence 12(7), 629-639 (1990). 